\title{
Ueber das positive centrale Scotom und über die Ursache der Sehstörung bei Erkrankungen der Netzhaut.
}

\author{
Von \\ Dr. Th. Treitel \\ Privatdocent in Königsberg i. Pr.
}

Bei meinen Untersuchungen der Functionsstörungen bei Amblyopien, über die ich zum Theil in der Arbeit „Ueber Hemeralopie und Untersuchung des Lichtsinnes“*) berichtet habe, wandte ich von vornherein meine besondere Aufmerksamkeit auf die centralen Scotome. Ich ging von der Ansicht aus, dass ein principieller Unterschied bestehen müsse zwischen der Art and Weise, in der die Abnahme des Sehvermögens bei Netzhaut-**) und bei Sehnervenkrankheiten zu Stande kommt. Hierfür spricht schon allein die auffallende Thatsache, dass nur bei Sehnervenleiden die Anomalie des Farbensinnes in der bekannten Skala Grün-Roth-Blau auftritt. In das

*) Diese Abtheilung des Archivs, p. 139-176.

**) Als Netzhautkrankheiten werden hier anch solche Affectionen des Angenhintergrundes bezeichnet, die nicht primär in der Retina ihren Sitz haben; die Sehstörungen bei allen Leiden des Angenhintergrundes beruhen ja, abgesehen von Trübungen der brechenden Medien, auf Veränderungen der Netzhaut. 
Wesen dieses Unterschiedes durfte ich hoffen, leichter eindringen $\mathrm{zu}$ können, wenn joh von vornherein einen begrenzten Theil des Gesichtsfeldes besonders ins Auge fasste.

Bei einem Vergleich der Retinal- und der Sehnervenscotome muss an erster Stelle folgende wesentliche Differenz hervorgehoben werden: die ersteren machen sich den Patienten ron selbst als dunkle Stellen bemerkbar, während die letzteren erst durch eine eingehende Prüfung des Gesichtsfeldes vom Arzte aufgefunden werden*). Wir bezeichnen nach Forster, welcher hierauf zuerst die Aufmerksamkeit gelenkt hat**), diese als negative, jene als positive Scotome.

Die von dem genannten. Forscher gegebene Erklärung stellt die positiven Scotome als eine Folge von Abstumpfung: des Lichtsinnes d. h. in seinem Sinne von Nachtblindheit hin. „In dem Augenblicke, wo die Kranken blinzeln, wo also für einen Moment die Netzhant von einem Halblicht getroffen wird, oder, wo sie auf eine schwach erleuchtete Fläche sehen, fällt auf dem erkrankten Theile der Netzhaut die Empfindung aus, und dadurch erscheint das Scotom dunkel."

Meine Beobachtungen $* * *$ ) stimmen mit denjenigen Förster's nicht überein; sie zeigen ohne Ausnahme, dass die Kranken das positive centrale Scotom bei hellem Tageslicht deutlich sehen und zwar nicht zeitweise, sondern

*) Ein Ausnahmefall von positivem Scotom bei einem Patienten mit Opticusatrophie, den ich früher mitgetheilt habe, vermag die Regel nioht umaustossen.

**) Bericht über den Ophthalmologen-Congress in Heidelberg: 1871, Zehender's klin. Monatsbl. 1871, pg. 337-345.

***) Hierbei sind auch solche Fälle mitgerechnet, bei denen das Scotom nicht genau central sass; es ist dies um so mehr gestattet, als oft ein anfänglich paracentrales positives Scotom sich im weiteren Verlauf über den Fixirpunkt ausbreitet, oder das umgekehrte Verhalten im regressiven Stadium eintritt, 
fortwährend. In den Lehrbüchern findet sich durchgehends die Förster'sche Anschauung, vielfach mit seinen eigenen Worten, so dass die betreffenden Autoren eigene Beobachtungen fur die Darstellung der in Rede stehenden Erscheinung kaum verwerthet haben düften. Soviel ich sehe, macht $W$ ecker*) allein eine Ausnahme; er bemerkt, dass der grauliche Fleck sich besonders deutlich zeigt, wenn die Patienten auf eine weisse Fläche sehen.

Die Ergebnisse meiner Untersuchungen erscheinen mit der von Förster gegebenen Erklärung des positiven Scotoms nicht vereinbar. Käme dieses zu Stande in Folge von Hemeralopie, so könnte es bei heller Beleuchtung nicht sichtbar sein; denn die Erscheinungen der Nachtblindheit machen sich nur bei herabgesetzter Beleuchtung bemeríbar.

Ferner steht der Umstand, dass das positive Scotom von den Patienten als grauer, nicht als schwarzer Fleck und von denselben Patienten je nach dem Stadium der Krankheit als verschieden intensiv grau bezeichnet wird, mit der Förster'schen Hypothese nicht im Einklang. Aus dem citirten Satz des genannten Autors folgt, falls ich denselben richtig verstanden habe, dass das Scotom den Kranken stets tief schwarz erscheinen muss. Denn dasselbe soll dadurch entstehen, dass die Empfindung in dem erkrankten Theile der Netzhaut ausfällt. Ein empfindungsfähiges, durch objectiven Reiz nicht erregtes Auge vermittelt aber die Empfindung von sehr dunklem Sehwarz.

Drittens stimmen die Anomalien der Farbenempindung mit der Förster'schen Erklärung nicht überein. Wäre diese richtig, so müssten bei einer Beleuchtung, bei welcher das Scotom sich deutlich markirt, Pigmente im Bereiche des letzteren ebenso wie von Hemeralopen bei herabgesetzter Beleuchtung bezeichnet werden. Diese erkennen Gelb und Grün besser als Blau und Roth, welche

*) Handb. von Graefe-Saemisch IV. pg. 623. 
sie schwarz nennen. Dass die Veränderungen des Farbensinns bei positiven Scotomen hiervon durchaus verschieden sind, wird später ausführlich dargelegt werden.

Endlich ist noch anzuführen, dass positive Scotome auch bei solchen Amblyopien beobachtet werden, die nicht mit symptomatischer Nachtblindheit einhergehen, nämlich bei Apoplexien der Retina, bei retinitis apoplectica und bei retinitis albuminurica. Die wesentlichen Merkmale des positiven Scotoms sind bei diesen. Krankbeiten aber nicht andere, als bei denjenigen, welche ausser Amblyopie auch Hemeralopie bewirken.

Nachdem sich in mir die Ueberzeugung von der Unhaltbarkeit der bisher gangbaren Erklärung des positiven Scotoms ausgebildet hatte, war ich bemüht, eine andere an deren Stelle zu setzen. Dabei bin ich zu der Anscharung gekommen, dass das positive Scotom eine entoptische Erscheinung ist, dass die Kranken den Schatten ihrer getrübten Netzhaut sehen.

Dass die Durchsichtigkeit der Retina bei denjenigen Krankheiten, bei denen man positive Scotome beobachtet, leidet, ist durch opthalmoskopische und pathologiseh-anatomische Untersuchungen bewiesen.

Für die Auffassung, dass durch "den Schatten dieser Trübungen die positiven Scotome entstehen, sprechen erstens die Bedingungen, unter denen die Patienten den grauen Fleck am deutlichsten seben, und zweitens Versuche, betreffend die Parallaxe des Schattens.

Was die erste Gruppe der Beobachtungen anbetrifft, so darf es als bekannt vorausgesetzt werden, dass man selbst kleine Opacitäten der Netzhaut*), ebenso wie des

*) Donders hält es nicht für ausgeschlossen, dass die granulirten Schichten der normalen Netzhaut entoptisch beobachtet werden können. (Die Anomalien der Refraction und Accommodation, übersetzt von Becker, Wien 1866 pr. 167.) Snellen und Landolt ist dies für die Nervenfaserschicht der gesunden Retina gelungen. (Handb. von Graefe-Saemisch, III. pg. 179.) 
hinteren Glaskörperabschnittes ohne Zuhülfenahme künstlicher Mittel entoptisch wabrnehmen kann und zwar wegen der geringen Entfernung des schattengebenden Körpers von der den Schatten auffangenden Stäbchenschicht.

Die normalen mouches volantes erscheinen deutlicher als mit blossem Auge, wenn man durch eine in einem undurchsichtigen Schirm angebrachte Oeffnung gegen eine helle Fläche blickt. Derartige Versuche haben, wie von vornherein wahrscheinlich, bei Patienten mit positivem Scotom für die in Rede stehende Frage keinen Aufschluss ertheilt: die Patienten sehen das Scotom ohne Weiteres scharf, so dass sie die Grenzen desselben genau angeben können.

Wichtige Resultate dagegen erhielt ich, als ich die Helligkeit festzustellen suchte, bei welcher die Patienten das Scotom am deutilichsten erkennen; es zeigte sich, dass dies bei derselben Beleuchtung der Fall ist, bei welcher die normalen und krankhaften Glaskörpertrübungen am deutlichsten wahrgenommen werden.

Bezüglich des Einflusses der absoluten Helligkeit auf die Schärfe der mouches volantes habe ich im Laufe des letzten Jahres, seitdem ich auf die Idee kam, dass das positive Scotom ein Schatten der dunklen Netzhant sei, wiederholt Beobachtungen angestellt; stets sah ich sie am genauesten bei Tageslicht von mittlerer Helligkeit, gleichviel ob mit oder ohne feine Oeffnung. Dies zeigte sich z. B. sehr klar, wenn ich erst gegen den bellen Himmel, dann auf das dunkle Dach eines meinem Arbeitszimmer gegenüber gelegenen Hauses und schliesslich auf das braungelbliche Haus selbst durch ein kleines Loch in schwarzem Papier blickte.

Die mouches volantes zeigten sich zuerst sehr deutlich im hellen Gesichtsfeld, verschwanden dann ganz, indem das letztere sich verdunkelte, und erschienen schliesslich 
sehr versehwommen bei schwach erleuchtetem Gesichtskreise.

Bei 10 Patienten habe ich den Einfluss der absoluten Helligkeit auf die Deutlichkeit des positiven Scotoms geprüft.

Ich liess sie dasselbe vergleichen einmal auf heller Papierbogen in constanter Entfernung; dann beim Blick gegen den Himmel und das schon genannte Haus resp. den von diesem entworfenen Schatten. Endlich variirte ich die Helligkeit durch Verdunkelung des Untersuchungszimmers, während die Patienten, den Rücken dem Fenster zugewandt, einen bestimmten Abschnitt der Jager'schen Schriftscalen betrachteten. Stets war das Resultat dasselbe: das Scotom erschien den Patienten am deutlichsten bei heller Beleuchtung.

Die genauesten Angaben machte Herr T., 28 Jahre alt, mit doppelseitiger abgelaufener chorioretinitis disseminata mit äusserst zahlreichen schwarzen Plaques, die auf dem rechten Auge auch die Gegend der macula lutea bedecken. Die Sehschärfe seines linken Anges ist ganz normal, die des rechten ohne Glas = Finger in $9^{\prime}$, mit $-3 \mathrm{D}$ $\mathrm{S}=5 / 24$; Jäger 3 wird in $8^{\prime \prime}$ langsam gelesen. Wenn der Patient bei Tageslicht in Leseweite mit dem rechten Ange den ersten Buchstaben des Wortes ,schlummern" in Jäger 6 Zeile 4 fixirt, so bedeckt ein dunkelgrauer Fleck dieses Wort vom "n" an und das nächste, "könnte", gänzlich; die Buchstaben schimmern durch das Scotom nicht hindurch; dasselbe hat die Form eines Dreiecks mit einer hellen, central gelegenen Stelle; es erscheint scharf begronzt bei Tageslicht, ganz verschwommen, sobald das Zimmer durch Herablassen der Jalousie mässig verdunkelt ist. Der sehr aufmerksame Patient giebt spontan an, bei heller Beleuchtung den Fleck so scharf za sehen, dass er ihn genau aufzeichnen könne, bei herabgesetzter sei er es nicht im Stande. 
Diese Bemerkung entspricht vollkommen meinen eigenen Wahrnehmungen bei den Untersuchungen des Einflusses der Helligkeit auf die mouches volantes.

Ganz dieselben Angaben machen auch Patienten mit circumscripten Glaskörpertrübungen: sie sehen dieselben am deutlichsten bei hellem Tageslicht.

Die abgesehen von der Beweglichkeit in den wesentlichsten Punkten übereinstimmenden Sehstörungen, über welche Patienten mit begrenzten Glaskörperopacitäten und Patienten mit positiven centralen Scotomen klagen, waren es, die mich zuerst daran denken liessen, dass die letzteren eine entoptische Erscheinung darstellen.

Bei beiden finden sich die gleichen Angaben über verschieden dunkles Grau der Scotome, über die eventuelle Möglichkeit, durch dieselben hindurch Objekte, z. B. Buchstaben schimmern zu sehen, über eine bestimmte, bei versehiedenen Kranken sehr wechselnde Gestalt des Scotoms und über die Abnahme der Deutlichkeit desselben bei herabgesetzter Beleuchtung.

Die letztere Erscheinung beruht auf der Eigenschaft unseres Auges, Helligkeitsdifferenzen am besten bei diffusem Tageslicht unterscheiden zu können*). Die Unterschiedsempfindlichkeit nimmt nicht allein bei geringerer, sondern auch bei erheblicherer Helligkeit ab; hiermit möchte ich die Angabe zweier Patienten erklären, dass sie das positive Scotom beim Blick auf eine von der Sonne beleuchtete Fläche weniger deutlich als bei Tageslicht sehen.

Für meine Erklärung des positiven Scotoms spricht ferner die Beobachtung, dass die Patienten relativ dunkle Scotome bei herabgesetzter Beleuchtung noch wahrnehmen, wenn auch verschwommen, während sie hellere unter denselben Verhältnissen nicht mehr erkennen. Denn der Schatten muss sich bei gleichen Graden von verminderter

*) Aubert im Handb. von Graefe-Saemisch, II. pg. 492. 
Helligkeit um so leichter bemerkbar machen, je dunkler er ist. So erkannte z. B. der mehrfach genannte Kr nke, der bei Tageslicht selbst die Buchstaben von Jäger 10 durch das Scotom nicht hindurchsehimmern sah, dasselbe bei mässig verdunkeltem Zimmer; andere Patienten, die bei Tageslicht durch den granen Fleck die Striche z. B. ron Jäger 1 wahrnahmen, sahen denselben bei gleichor Abnahme der absoluten Helligkeit nicht mehr. Dem entsprechend erkannte ein Patient mit Glaskörpertrübungen, der beim Blick gegen den bellen Himmel zahlreiche grane bewegliche Fäden nnd drei dunklere Pankte sah, nur die letzteren, wenn er auf die hintere Wand des Zimmers sein Auge richtete.

Die Deutlichkeit, mit der wir ein Object wahrnehmen, ist ausser von der absoluten Helligkeit und dem Helligkeitsunterschied von der Grösse abhängig. Dem entsprechend werden im regressiven Stadium kleiner gewordene Scotome weniger scharf als früher, bei grösserem Umfange, wahrgenommen. Dabei ist jedoeh zu berucksiehtigen, dass der Wleck gleichzeitig heller als vordem erscheint. Es bleibt somit unentschieden, wieviel die Verkleinerung und wieviel die Aufhellung der Retinatrübung dazu beiträgt, um das Scotom weniger deutlich erscheinen zu lassen.

Die zweite Versuchsreihe betrifft die parallaktische Bewegung des Scotoms.

Um diese nachzuweisen, stellte ich mit einigen Patienten Beobachtungen nach der Methode an, welche Helmholtz*) als zweite zur entoptischen Wahrnehmung der Netzhantgefasse beschreibt. Sie besteht darin, dass das zu prüfende Ange auf einen dunklen Hintergrund blickt, und unterhalb oder seitlich vom Auge ein brennendes Licht hin und her bewegt wird. Die Netzhautgefässe erscheinen dabei sehr gross, weil als Lichtquelle das

*) Physiol. Optikx, pg. 158. 
Netzhautbild der Flamme dient, und die Lichtstrablen somit stark divergent auf den schattengebenden Körper fallen.

Die genauesten Angaben machte der oben genannte Herr T. Zuerst zeigte ich $\mathrm{ihm}$, wie man die eigenen Netzhautgefässe erkennen könne; er sah bei einem Versuche, den er in meiner Gegenwart mit seinem functionell normalen linken Auge anstellte, sofort die Purkinjé'sche Aderfigur; er beschrieb dieselbe ganz richtig, auch die Richtung, in der die Gefässe sich bei horizontaler und bei vertikaler Bewegung des Lichtes verschieben. Er zeichnete nach mebrmaligen Beobachtungen die Figur auf und markirte dabei den halbmondförmigen Schatten des Randes der Fovea centralis. Ich führe dies nur deswegen so eingehend an, um den Leser zu überzengen, dass die Angaben des Kranken über die entoptischen Erscheinungen als durchaus zuverlässig angesehen werden dürfen. Derselbe hat nun die gleichen Untersuchungen an seinem reehten Auge angestellt und zu wiederholten Malen Folgendes beobachtet. Die Gefässfigur erscheint durch einen grossen, graulichen Fleck unterbrochen, der dicht neben dem Fixirpunkt, nach rechts von demselben liegt, und dessen oberer und unterer Rand nicht zu erkennen sind. Wird das Licht unterhalb des Auges in horizontaler Richtung bewegt, so verschiebt sich die dunkle Stelle in gleicher Richtung, wie die Gefässe, ohne dass die Lage derselben zu einander verändert wird. Hiermit ist eine Parallaxe des positiven Scotoms festgestellt.

Der Patient gab ferner ganz spontan an, dass das Scotom bei dieser Untersuchung 15-20 Mal so breit, als die dicksten Gefässe, und etwa $10 \mathrm{Mal}$ so breit als bei Tageslicht erscheint, und dass die Ränder desselben viel verschwommener als bei Tageslicht aussehen. Die erste Erscheinung wird durch die sehr geringe Entfernung des 
Bildes der Flamme ron dem schattengebenden Körper, die zweite dadurch erklärt, dass der Schatten in Folge eines gewissen Umfanges des Netzhautbildes der Lichtquelle weniger scharf begrenzt ist.

Hiermit scheint mir die in Rede stehende Erilärung des positiven Scotoms bewiesen zu sein; es scheint mir, dass bei dieser Frage die zuverlässigen Beobachtungen eines einzigen Patienten, bei dem die Erscheinungen des positiven Scotoms mit den bei anderen Kranken beobachteten übereinstimmen, etwa einen gleichen Werth beanspruchen dürfen, wie Selbstbeobachtungen eines Untersuchers für die Pbysiologie des Auges.

In der Zeit, in der ich mich mit diesen Prufungen beschäftigte, fanden sich unter den Patienten mit positivem Scotom noch dreit andere, mit denen ich sie glaubte anstellen zu können. Sie machten ebenfalls mehrmals positive Angaben über eine Bewegung des Scotoms bei Verschiebung des Lichtes. Ihre Angaben waren aber nicht so eingehend und erschienen mir nicht so vollkommen überzeugend, wie bei dem oben genannten Herrn, so dass ich sie erst an zweiter Stelle und obne weitere Details anfiubre.

Endlich sehien es mir geboten, zu versuchen, ob die Patienten bei der Brewster-Donders'schen Methode das Scotom doppelt sehen, bei welcher der Beobachter durch zwei kleine, nahe an einander liegende Oeffnungen einer undurchsichtigen Platte gegen eine helle Fläche blickt. Den Versuch, auf diesem Wege die Schattennatur des positiven Scotoms nachzuweisen, musste ich aufgeben, als ich mich bei oft wiederholten Beobachtungen davon überzeugt hatte, dass die Schatten der Retinalgefässe auf diesem Wege nicht verdoppelt werden können. Wenn ich nämlich ein Blatt Papier mit zwei kleinen, 2,5 Mm. von einander entfernten Lochern vor meinem Auge hin und her bewegte, sah ich die sehr zierlichen Gefässe stets in 
derselben Weise, als wenn ich nur durch ein Loch blickte. Die Gefässe liegen demnach zu nahe an dem Stäbehen, um bei dieser Art des Versuches zwei zu differenzirende Schatten zu entwerfen; um so unwahrscheinlicher war dies für die viel grösseren Scotome.

Auf Grund der hier vorgetragenen Erklärung des positiven Scotoms kann man die Eigenschaften, die denselben nach den Angaben der Patienten anhaften, ohne Zwang erklären.

So macht eine verschieden saturirte Netzhauttrübung eine verschiedene Dunkelheit der Scotome verständlich. Dass die graue Nüance der Schatten nicht immer dieselbe ist, geht erstens aus den Angaben herror, welche die Patienten in den einzelnen Stadien des Leidens machen; sie bemerken meist als erstes Zeichen der Besserung*), dass ,sie deutlicher durch den Flecken hindurchsehen können", dass "der Schatten sich aufhellt". Zweitens erscheint gelegentlich Patienten mit doppelseitigem Scotom dasjenige auf dem einen Auge dunkler als auf dem anderen. Die meisten beschreiben, wie schon oben erwähnt, das Scotom als einen grauen Fleck, als einen "Schatten", der an dem fixirten Gegenstand haftet, und durch den der letztere mehr oder weniger deutlich hindurchschimmert. Ihnen erscheint das Scotom auch dann nicht schwarz, sondern dunkelgrau, wenn kleine weisse Papierquadrate in Leseweite nicht wahrgenommen werden: die Trübung der Retina ist durchscheinend; hierfü spricht auch die Beobachtung, dass oft der Schimmer grösserer Quadrate noch erkannt wird, obwohl diese in der betreffenden Entfernung kleiner als das Scotom sind. Dass die Retina jemals so undurchsichtig werden sollte, um gar kein Licht passiren $\mathrm{zu}$ lassen, dürfte nicht wahrscheinlich sein. Sollte dieser Fall

*) Seltener kündigt sich das regressive Stadium durch eine Verkleinerung des Scotoms an. 
eintreten, so müsste das Scotom in der That schwarz erscheinen, selbstverständlich nur so lange, als die Erregbarkeit der percipirenden Elemente im Scotom orhalten bleibt; denn sobald diese erlischt, wird das Scotom negativ, ebenso wie der normale blinde Fleck.

Eine ungleichmässige Trübung der Netzhaut erklärt die Beobachtung, dass das Scotom mitunter nicht gleichmässig grau erscheint, dass sich in demselben, wie manche Patienten angeben, kleine helle Stellen markiren. Der oben genannte Herr T. sah in der Mitte des Schattens eine ganz klare Partie, ziemlich viele Patienten erkannten den Schimmer eines weissen Papierquadrates von bestimmter Grosse nur in einem Theil des Scotoms.

Ferner wird aus der Eigenfarbe der Retinatrübung eine bestimmte Farbung des Scotoms verständlich. Es ist allgemein bekannt, dass positive Scotome in Folge von Netzhautblutung anfangs röthlich, später gelblich oder grünlich erscheinen können. Bei zwei Patienten begegnete ich der Angabe, dass sie das Scotom ausgesprochen blau sehen. Der eine von ihnen, der an recidividirender Chorioretinitis maculae luteae bei Myopia excessiva leidet, erkennt als erstes Symptom eines Rückfalls einen blauen Fleck auf hellen Gegenständen z. B. auf der Kuppel einer brennenden Lampe*).

Endlich erklärt sich auf die genannte Weise eine bestimmte Form des Scotoms**), die von den Kranken stets angegeben wird; sie erkennen dieselbe, so viel ich gesehen habe, am bestem auf bedrucktem Papier, z. B. an den Jäger'schen Leseproben und bezeichnen genau den rom Scotom bedeckten Theil eines bestimnten Abschnittes. Wenn man die Untersuchung in dieser Weise anstellt, so kann man sich gleichzeitig über den Grad der Sehstörung

*) Ophthalmoslzopisch zeigten die entzündichen Heerde der Macula keine anfallende Färbung.

**) Die Grösse des Scotoms schwankte innerhalb sehr weiter Grenzen. 
orientiren, indem man die Kranken angeben lässt, an welchen Nummern sie die Buchstaben resp. deren Striche durch das Scotom erkennen. Der Schatten wird meistens als rundlich bezeichnet, entsprechend der Form der ophthalmoskopisch sichtbaren Exsudate, sein Rand oft nicht als regelmässig, sondern ausgezackt. Es kommen aber auch andere, mehr oder weniger regelmässige Formen zur Beobachtung, z. B. dreieckige, wie schon oben erwähnt ist. Eine Patientin mit Chorioretinitis maculae luteae bei excessiver Myopie verglich das Scotom auf dem Höhestadium des Processes mit einem Eichenblatt. Die Dame konnte, als ich sie das erste Mal sah, mit dem rechten Auge noch kleinsten Druck lesen; das Scotom war reehts vom Fixirpunkt; es zog sich bald über diesen, so dass von Jäger 5 nur kleine Worte mühsam entziffert wurden. Nach vierwöchentlicher Behandlung in meiner Klinik war die Macula frei, kleiner Druck wurde erkannt, das Scotom sass wieder rechts vom Fixirpunkt und hatte eine unregelmässige Gestalt angenommn. Ausdehnung und Lage der durch Netzhautblutungen hervorgerufenen Scotome entsprechen dem ophthalmoskopischen Bilde derselben; ein Gleiches gilt von den durch Fremdkörper der Retina bedingten Scotomen.

Ich gehe jetzt zur Besprechung der Anomalien des Farbensinnes bei positiven Scotomen über, soweit sie bei der Untersuchung mit Pigmenten zu Tage treten. Eine eingehende Würdigung derselben erscheint mir um so mehr gerechtfertigt, da die Farbensinnstorungen der Netzhautkrankheiten bei dem vorwiegenden Interesse für diejenigen der Sehnervenleiden bisher nur wenig berucksichtigt worden sind.

Es liegen mir Beobachtungen von 60 Kranken ror, von denen 21 auf die letzten 15 Monate kommen. Diese 21 Patienten sind mit weissen, blauen, gelben, rothen und grünen Quadraten von 2,3,5,10 und $20 \mathrm{Mm}$. Seite auf 
schwarzem Grunde, die übrigen nur mit 5 and 20 Mrn. Quadraten geprufft worden; die letzteren ausschliesslich am Förster'schen Perimeter, jene auch in Leseweite, ein Modus, der namentlich bei Kurzsichtigen vorzuziehen ist. Ausserdem stellte ich im letzten Jahre bei den meisten Kranken die grösste Entfernung fest, in welcher die kleinsten noch erkennbaren Farbenquadrate im richtigen Tone benannt wurden.

Die Pigmente sind Heidelberger Blumenpapiere, dieselben Nüancen, die ich bei meinen Gesichtsfelduntersuchungen angewandt habe. Ausserdem wurden die bezeichneten 21 Patienten mit der Bull'schen chromatoptometrischen Tabelle in 1 Meter Distanz geprüft. Die Heidelberger Pigmente wurden entweder von der Peripherie nach dem Centrum verschoben oder sogleich in der Richtung der Gesichtslinie gezeigt*), ohne dass sie vorher gesehen werden konnten. Im letzteren Fall crhalt man zuverlässigere Angaben über die Veränderungen der Pigmente im Scotom, denn es ist für die meisten Patienten, wenn sie die Farbe einmal kennen, nicht leicht, unbefangene Angaben zn machen. Bei einseitig Erkrankten erseheint ein Vergleich mit dem gesunden Auge, selbstverständlich unter sonst gleichen Bedingungen, sehr empfehlenswerth; man kann auf diese Weise Functionsstörungen der Macula lutea, d. h. centrale Scotome im weitesten Sinne des Wortes, nachweisen, die bei einem Vergleich mit den extramaculären Parthien desselben Auges nicht zu Tage treten.

Bei der Untersuchung mit den Heidelberger Pigmenten erschienen der Mehrzahl der Kranken die Farben im Scotom verschleiert, während der Ton richtig erkannt wurde. Einige nahmen die Verdunkelung fur Blau oder Roth deutlicher als für Grün und Weiss wahr. So

*) Die meisten Patienten mit positivem Scotom sind noch im Stande, central zu fixiren odex wissen anderenfalls, ob sie das Untersuchungsobject direct oder indirect betrachten. 
gab ein Patient mit einer Blutung an der Macula bei retinitis apoplectica an, dass ihm central nur Blau und Roth unklarer erschien, ein anderer mit doppelseitigem grossen centralen Scotom in Folge von retinitis albuminurica ohne Blutung an der Macula fand Weiss relativ am wenigsten verschleiert. Aehnliche Beobachtungen wurden öfter gemacht: eine Patientin mit chorioretinitis maculae luteae bei hochgradiger Myopie konnte im Scotom die Form der 5 Mm. Quadrate nur bei Weiss erkennen und sah Blau mehr als Grün und Roth beschattet. Im Ganzen kam es aber selten vor, dass das Scotom nur mit den Pigmenten nachgewiesen werden konnte, während Weiss central deutlicher als peripher erschien.

Dieser Gruppe steht eine zweite, kleinere, von 7 Fällen gegenüber, bei denen im Scotom keine Farbe im richtigen Tone erkannt wurde. Die Pigmente unterschieden sich aber durch verschiedene Helligkeit, und zwar ersehien Grịn und Gelb heller als Roth und Blau.

Als Beispiel führe ich folgenden, auch klinisch interessanten Fall von Thrombose der vena centralis retinae an.

Die 30jährige Kranke stellte sich mir am 31. December 1883 mit der Angabe vor, seit sechs Monaten auf dem rechten Auge schlechter zu sehen. Die wesentlichen Veränderungen des Augenhintergrundes waren starke Sehlängelung und Verdickung der Venen bei wenig verengten Arterien; die Yenen an wenigen Stellen unterbrochen, auf der Höhe der Bogen fast schwarz; die Retina in der Umgebung der Papille sehr trübe und mässig verdickt, die ganze Papille blutig gefärbt; diese Färbung lüst sich im aufrechten Bilde in zahlreiche kleine rothe Flecke auf, die mehr auf als in der Papille zu liegen scheinen; die letztere dabei nicht geschwellt, noch durchscheinend, zahlreiche kleine Gefässe erweitert; in der Retina neben dem temporalen Rande des Sehnerven einzelne kleine weissliche Plaques. An der Macula eine ovale Blutung von $1 / 3$ Papillengrösse. in $4^{\prime}$.

Dem entsprechend ein centrales Scotom mit $\mathrm{S}=$ Finger

v. Graefe's Arehiv für Ophthajmologie, XXXI. 1. 
Die Farben der 20 und 10 Mm. Quadrate werden

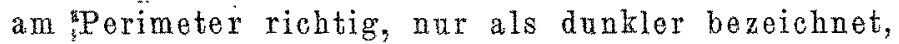
der 5 Mm. Quadrate, welche neben dem Scotom richtig erseheinen, nicht wahrgenommen: grün und gelb $=$ weiss, blan $=$ sehwarz, roth $=$ dunkel. Aussen- und Farbengrenzen normal,

Nach dreiwöchentlicher klinischer Behandlung erkannte die P. einzelne. Buchstaben von Jäger 10 und die Farben der $5 \mathrm{Mm}$. Quadrate; die. Blutung an der Macula weniger dunkel.

Die Anzahl der Fälle, die kein Pigment erkannten, wird übrigens grösser, wenn man aus der ersten Gruppe diejenigen hierher rechnet, denen ein. Theil des Quadrates grau erscheint. Es ist klar, dass die Farbe kleinerer Objecte überhaupt nicht erkannt worden wäre.

Die Anomalien des Farbensimes der ersten und zweiten Gruppe sind nur graduell von einander unterschieden. Dafür spricht erstens die Thatsache, dass die vordem nicht erkannten Pigmente bei unverändertem Gesichtswinkel richtig benannt werden, sobald der krankhafte Process, vie die Verbesserung der Sehschärfe und der ophthalmoskopisch sichtbaren Veränderungen zeigt, ins regressive Stadium eintritt; zweitens die Beobachtung, dass in einer Zeit, in der kleinere Pigmente farblos erscheinen, grössere sich in richtigem Tone darstellen, trotzdem sie ganz rom Scotom bedeckt werden. Dies demonstrirt schon der eben mitgetheilte und mag auch noch folgender Fall orläutern; derselbe erseheint ferner deswegen interessant, weil or ein positives centrales Scotom bei fast normaler centraler Sehschärfe und dem entsprechend wenig veränderter macula latea zeigt.

Herr J., 42 Jahre alt, leidet an einer luetischen Retinitis (Jacobson) des linken Auges; die Retina in der Umgebung der Papille ist schwach getrübt und geschwellt, die Contouren der letzteren in geringem Grade verwaschen, ihre Oberfäche ganz normal, an der Macula keine circumscripte Veränderung. o. d. vollkommen gesund. Roseola, Geschwüre im Rachen, 
condylomata ad anum liessen an der Diagnose kënen Zweifel aufkommen.

20. März 1880. L. Mp. $0.75 \mathrm{D} \mathrm{S}=6 \% 60$; Aussen- und Farbengrenzen normal; kleines positives centrales Scotom, in dem auf 1' Entfernung die Farben der $20 \mathrm{Mm}$. Q. verschleiert, der $5 \mathrm{Mm}$. Q. farblos erscheinen; die Ecken der $20 \mathrm{Mm}$. Q. sind wie abgeschnitten, die $5 \mathrm{Mm}$. Q. sehen ganz nnregelmässig aus. Nach einer Schmier- und Schwitzkur am

3. April das Scotom kleiner und klarer, Farben der $5 \mathrm{Mm}$. Q. in 1' richtig erkannt, nur beschattet, Form der Q. ebenfalls richtig. $\mathrm{M}_{\mathrm{p}}$. $0.75 \mathrm{D} \mathrm{S}<{ }^{6} / 6$.

13. April, $S=6 / 6$, Scotom nicht mehr nachzuweisen.

In seltenen Fällen geht im Scotom die Empfindung für farbige und weisse Objecte von bestimmter Grösse vollkommen verloren.

Fin 67jâhriger Herr mit einer Blutung an der Macula erkannte bei normaler Aussen- und Farbengrenze ${ }^{*}$ ) im Scotom nur die obere Hälfte der $20 \mathrm{Mm}$. Q, $5 \mathrm{Mm}$. Q. uberhaupt nicht.

Folgender Fall ist deswegen sehr wichtig, weil er zeigt, dass bei positiven Scotomen eine auf der Höhe des Processes vollständig fehlende Empfindung sich ganz oder fast ganz normalisiren kann.

Herr H., 20 Jahre alt, wird am 21. Februar 1884 mit einer floriden chorioretinitis disseminata oc. sin. in meiner. Klinik aufgenommen. o.d. vollkommen gesund.

L. $\mathrm{S}=$ Finger in $6 ; 2-10 \mathrm{Mm}$. Q. werden central in 7" gar nicht gesehen; Patient giebt spontan an, die Farben selbst der $2 \mathrm{Mm}$. Q. bei geringen Drehungen des Kopfes erkennen zu können. Farben der $20 \mathrm{Mm}$. Q. central erkannt.

18. April 1884. E. $\mathrm{S}>^{\mathfrak{5} / 50}$; Farben der $2 \mathrm{Mm}$. Q. in $7^{\prime \prime}$ richtig; Scotom heller und kleiner.

*) Alle Gesichtsfelduntersuchuigen sind wie die früher von mir veröffentlichten mit $20 \mathrm{Mm}$. Quadraten auf schwarzem Grunde am Förster'schen Perimeter ausgeführt. 
Bei diesem Patienten ist leider nicht notirt, ob das Scotom als grau oder schwarz bezeichnet wurde; der mehrfach genannte Herr T. konnto im Scotom den Schimmer eines reissen $10 \mathrm{Mm}$. Quadrates kaum wahrnehmen; er bezeinhnete den Fleck als dunkelgran und erkannte in demselben den Schein einer Flamme.

Is folgt jetzi eine dritte Gruppe von Fälen, die zwischen der ersten und zweiten steht, und dadurch ansgezeichnet ist, dass nur einzelne Farben richtig benannt werden. Sie kann in zwei Abtheilungen getrennt werden: die Fälle des einen begehen Verwechslungen zwischen Blau und Grün, die der anderen seber alle Pigmente bis auf Roth farblos. Von dieser letzten Kategorie habe ich nur arei Fälle*) beobachtet; dieselben einzeln aufzuführen, scheint mir deswegen geboten, weil sie gleichzeitig boweisen, dass die in getrennten Gruppen anfgeführten Anomalien des Farbensinnes verschiedenen Stadien desselben Leidens entsprechen.

Enil N., 32 Jahre alt, zeigt auf dem rechten Auge an der Dacula ein grosses Exsudat von grüulicher Farbe; Ursache nicht nachruweisen. Aussen- und Farbengrenzen normal bis auf die Grüngrenze, die an einer Stelle rom Scotom durchbrochen ist. Sehr grosses centrales Scotom firr $20 \mathrm{Mm}$. Q., in dem Roth richtig, Blau und Grün granweiss erscheinen. $\mathrm{S}=$ Finger in 14: Nach 4 Wochen Hm. ${ }^{1 / 14} \mathrm{~S}<{ }^{20} / 40 ; 20 \mathrm{Mm}$. Q: Roth: röthlich, Blau: dunkelgrün, Grün: grau.

Herr G., 36 Jahre alt, wird mit einer typischen chorioretinitis luetica (Förster) ocul. utriusque in meiner Klinik am

1. December 1883 aufgenommen. R. $8=$ Finger in 5 ; sehr grosses Scotom; $20 \mathrm{Mm}$. Q.: weiss und Farben: gran;

*) Hiermit ist die Zahl der Individuen, nicht der untersucbten Angen bezeichnet. Uebrigens geben die $Z$ ablen nui eine ungefähe Vorstellung von dem Verhaltniss der verschiedenen Gruppen, weis einzelne Patienten in mehreren onftreten: bei diesen konnten mit verschieden grossen Pigmenten zla derselben oder mit gleich grossen zu verschiedenen Zeiten mehrere Arten der Farbensinnstörung: nachgewiesen werden. 
das Q. als solches nur bei Weiss und Grün erkannt; es erscheint dabei kleiner als peripher. Ord. Dunkelzimmer, Schmierund Schwitzkur.

20. Decmber 1883. $S=$ Finger in $14^{\prime}$; Jäger 9 in $8^{\prime \prime}$ einzelne Worte; Scotom viel kleiner. $20 \mathrm{Mm}$. Q.: Roth zeitweise richtig, Blau dunkel, Grün weisslich; Form aller Quadrate erkannt.

28. Januar 1884. $S=$ Finger in $14^{\prime}$, Jäger 7 einzelne Worte; $20 \mathrm{Mm}$. Q. central Roth und Blau: richtig, nur beschattet, Grün: grau, im peripheren Theil des Scotoms: Roth: roth, Blau: bald grün, bald grau, Gruan: weiss.

13. Februar 1884. $S=5 / 60$, Jäger 5. $20 \mathrm{Mm}$. Q. central: Blau und Roth richtig. Grün und Gelb nicht erkannt; 10, 5 und $3 \mathrm{Mm}$. Q. ebens $0 ; 2 \mathrm{Mm}$. Q. Roth: roth, Blau: meist grün, selten blau, Gelb und Grün: grau.

Die Macula lutea des linken Auges ist von Fornherein aicht afficirt.

1. December 1883. L.: E. $\mathrm{S}<\%$, Jäger 2 in 17 ct.; $2 \mathrm{Mm}$. Q. Roth in 90 ct., Blau in 50 et., Grän in 25 ct., Gelb in 20 ct. richtig benannt. Aussengrenze normal; breites ringförmiges Scotom.

Bull'sche Tafel in 1 Meter

am 1. December 1883. L. N 4 erkannt, R. keine Farbe; einen Monat später R.: Blau, Roth und Grün richtig in N. 18; N. 14 keine Farbe; ebenso am 13. Februar 1884.

Frau B., 57 Jahre alt, stellte sich am 16. März 1882 mit der Angabe vor, seit acht Tagen auf dem rechten Auge einen grauen Fleck nach links vom Fixirpunkt zu bemerken; sie sei von jeher auf beiden Augen stark kurzsichtig, auf dem linken schwachsichtig gewesen.

R. Mp. $11 \mathrm{D} \mathrm{S}=5 / 36$, Jäger 3 in $6^{\prime \prime}$ gelesen. Wenn Patientin in dieser Entfernung in $J \ddot{a g} \theta \mathrm{r} 5, \mathrm{Z} \theta$ ile 1, das Wort , $\mathrm{s0}^{\circ}$ fixirt, dann ist das ganze vorhergehende Wort „,verab-. scheut" verdeckt.") Farben der 5 Mm. Q. in 6" richtig, am Q. fehlt die linke obere, zeitweise auch die linke untere Ecke. Ophth.: grosser, ringförmiger, ganz atrophischer conus,

*) Die Lesefähigkeit ist natürlich viel mehr beschränkt, wenn das Scotom nach rechts, als wenn es nach links vom Fixirpunkt sitzt. 
chorioretinitis macnlae luteae in der bekannten Strichform, ähnlich den Sprüngen einer lackirten Fläche.

Der Verlauf war bei dieser Kranken trotz aller nur denkbaren therapentischen Versuche sehr ungunstig. Die uber mehrere Jahre ausgedehnten Beobachtungen der Funktionsstörungen, speciell des Farbensinns, erscheinen sebr lehrreich.

1. April 1882. Scotom grösser, bedeckt den Fixirpunk ohne sichtbare frische Veränderungen der macula lutea. Jäger 5 nicht mehr erkannt.

8. Juli 1882. Mp. 11 D Finger in 6. Jäger 9 einzelne grosse Buchstaben; $5 \mathrm{Mm} . \mathrm{Q}$ im Scotom nur Roth erkannt.

5. Januar 1883. S. idem. $20 \mathrm{Mm}$. Q. in $7^{\prime \prime}$ Form und Farbe richtig, in der Mitte derselben ein dunkelgrauer Fleck, dureh den Roth und Grün hindurchsehimmert, Blau sehr undentlich, Gelb=weiss. $5 \mathrm{Mm}$. Q. in $7^{\prime \prime}$ Roth: roth, Grün: bald weiss, bald grün, Blau: bald blau, bald dunkel, Gelb: weiss; Q. als solches nicht erkannt.

27. November 1883. Ophth.: an der Macula eine grosse bräunliche Plaque, temporalwärts neben derselben eine frische umfangreiche Blutung. $S=$ Fingor in $2^{*}$, mit $-11 \mathrm{D}$ in $\dot{6}^{\prime}$; Jäger 15 einzelne Buchstaben. $3 \mathrm{Mm}$. Q. in $6^{\prime \prime} \mathrm{Roth}$ : röthlich, Grün und Gelb: weisslich, Blau: ,gar nicbts". Patientin erkennt die Farben, wenn sie das Ange nach der Nase dreht. Aussen- und Tarbengrenzen normal, blinder Fleck verbreitert. Bull'sche Tafel in 1 Meter ohne Glas nichts, mit $-11 \mathrm{D}$ in $\mathrm{N} .18$ einzelne Punkte, aber keine Farbe gesehen.

1. April 1884. Ophth.: Blutung ganz resorbirt, an ihrer Stelle erscheint der Hintergrund weisslich; Scotom grösser; $20 \mathrm{Mm}$. Q. in $6^{\prime \prime}$ keine Farbe erkannt.

14. December 1884. Stat. idem.

Diese Fälle zeigen, dass es sich um eine erheblichere Sehstorung handelt, wenn bej erhaltener Empfindung von Roth das blane und grune Pigment farblos, als wenn das blane gletis grü erscheint.

Bei der Untersuchung mit den Bull'schen Pigmenten wurde die Verwechslung von Blau und Grün viel häufiger als bei den Heidelberger Pigmenten 
beobachtet. Unter 17 Fällen, die ich mit der Bull'schen Tafel prafte, erkannten 4 entweder keine Farbe oder nicht einmal die Quadrate, 5 die Farben richtig, 8 begingen die fragliche Verwechslung*): sie bezeichneten Blau als grün und Grün als blau und gleichzeitig selbst kleinere Quadrate der Heidelberger Pigmente in richtigen Tone.

Bei fünf von ihnen konnte ein Vergleich mit dem zweiten gesunden Auge angestellt werden, das die Farben der Bull'schen Tafel bis incl. N. 1,5 richtig erkannte.

Herr H.,**) 20 Jahre alt, leidet auf dem linken Auge an florider chorioretinitis disseminata. Am 21. Februar 1884. L. $\mathrm{S}=$ Finger in $6^{4}$; Patient kann in 1 Meter die Quadrate der. Bull'schen Tafel nicht erkennen. R. E. $S=1 ; B$ ull N. 1.5 riehtig, N. 1 unsicher.

15. Oetober 1884. L. E. $\mathrm{S}<5 / 18 ; 2 \mathrm{Mm}$.Q. in 1 ' richtig, nur Gelb unsicher: Bull N. 18: Blau und Grün verwechselt, Gelb: weiss, Roth: roth, ebenso bis N.4; N, 3 alle Farben grün.

Herr B., 40 Jahre alt, zeigt ein cireumseriptes, weisslichgranes Exsudat an der Macula des rechten Auges bei sonst normalem Hintergrund.

L. E. $S=1$. Bull N1 richtig.

R. E. $S=5 / 18$. Aussen- und Farbengrenzen normal; Farben der $2 \mathrm{Mm}$. Q. in 1' Entfernung richtig; sie erscheinen bei einem Vergleich mit dem linken A uge dunkler und kleiner. Bull N. 18 Gelb und Roth, Grün und Blau sehen gleich aus; diese Q. werden bald blau bald grün genannt. Bbenso bis incl. N. 3.

Frl. M., 20 Jahre alt, ist seit ihrer Kindheit stark kurz-

*) Hierbei sind nur solche Fälle gezählt, welche Blau und Grün der saturirteren Nüancen (bis N. 8 incl.) mit einander verweehselten; bei den schwächeren Náncen bleibt es fraglich, ob es sich um eine charakteristische Erscheinung handelt, wenn Blau als grün und umgekehrt bezeichnet wird; bei der Mehrzahl meiner Patienten war dies schon in N.18 der Fall.

* D) Dieser Fall ist oben schon angefuhrt. 
sichtig and sielnt anf dem linken Ange seit drei Monaten schlechter. Ophth.: chorioretinitis maculae luteae.

R. Mp. $29 \mathrm{D} S=\mathrm{J}$ gager liebr. 22 in 5 heter. Bull N. 1,5 richtig mit $-16 \mathrm{D}$, o hne Glas in N. 18 keine Farbe.

L. Hip. $16 \mathrm{D} \mathrm{S}=$ Jäger 24 in 5 Meter. Bull N. 18 Gelb=Roth, Blan=Grün, bald blau, bald grün benannt, ebenso bis incl. N.3. Aussen- und Farbengrenzen normal; kleines positives centrales Seotom, in dem die $5 \mathrm{Mm}$. Q. in ricbtigem Tone, nur beschattet erscheinen.

Herr K., 18 Jahre alt; beiderseits abgelaufene chorioretinitis disseminata mit grossen schwarzen Plaques an der Macula.

L. Mp. $1 \mathrm{D}, \mathrm{S}=1 ; \mathrm{B}$ ull N. 2 richtig.

R. Mp. $1 \mathrm{D} S>5 / 18$; Bull N.11 richtig, N. 8 Blau =Grun; Farben der 2 Mm. $Q$. in 1 Meter erkant. Temporalwärts vom Fixirpunkt ein positives Scotom, in dem ein Theil der $20 \mathrm{Mm}$. Q. vollstandigg versehwindet.

Wenn ich nun die Ergebnisse meiner Untersuchungen तes Farbensinnes bei positiven centralen Scotomen zusammenfasse, so zeigt sich der geringste Grad von Störung darin, dass die Pigmente dunkler, wie von einem Schleier gedeckt, aber noch im richtigen Tone erscheinen. Dann folgt ein Stadium, in dem Blau als grün, Grün entweder als grïn oder grau bezeichnet wird, während Roth seine Farbe behält. Im weiteren Verlauf erscheint Blau und Grün grau, Roth noch roth, endlich auch dieses farblos. Die Pigmente unterscheiden sich dann nur durch verschiedene Helligkeit und zwar werden Roth und Blau dunkel, Grün und Gelb hell benannt. Schliesslich wird das Scotom entweder nur für die kleineren oder für alle von mir angewandten Quadrate absolnt d. h. der Schimmer selbst der weissen Objecte wird nicht mehr erkannt. In diesen Fallen bleibt aber die Empfindung für intensiveres Licht noch erhalten. Sobald diese erlischt, ist das Scotom negativ. Gegenuber den Bull'solien Pigmenten begehen die Patienten relativ oft Verwechslungen von Blau und Grün und bezeichnen nicht allein Blau als grün, sondern 
anch Grün als blau; sie sind meist im Ungewissen, ob sie diese Farben blau oder grün nennen sollen.

Diese Anomalien des Farbensinns lassen sieh als eine Folge des Schattens, der die Zapfen der kranken Netzhautparthie bedeckt, erklären. Der Sehatten bewirkt eine Herabsetzung der absoluten Helligkeit und demgemäss dieselben Störungen des Farbensinns wie abnehmende Beleuchtung für das normale Auge. Die letzteren sind bekanntlich von Aubert*) sehr eingehend studirt worden; er kam zu folgenden Resultaten:

1) Die I'igmente werden bei selır verminderter Intensität der Belenchtung farblos, unterscheiden sich aber noch dureh ihre Helligkeit. Auf schwarzem Grunde erscheint Roth am dunkelsten, dann folgt Blau, dann Hellgrün und Hellblau, dann Gelb und schliesslich Weiss, das bei weitem am hellsten aussieht.

2) Bei der schwächsten Beleuchtung kann Roth im richtigen Tone erkannt werden, erst bei stärkerer Grün und Hellblau, bei noch stärkerer Dunkelblau.

3) Die Pigmente verändern bei abnehmender Beleuchtung ihren Farbenton: auf schwarzem Grunde erscheint Roth als dunkles Braun, Gelb schmutzig grau mit einem röthlich gelbem Stiche, Hellgrün und Hellblau sehen gleich aus, volles Ultramarinblan wie graublaues Aktenpapier, Dunkelgrün schmutzig graublau.

Man sieht hieraus, dass die Anomalien des Farbensinnes bei positiven centralen Scotomen im Wesentlichen tabereinstimmen mit dem Farbensinn normaler Augen bei herabgesetzter Helligkeit. Auf diese Weise finden zwei Symptome eine Exklärung, die als charakteristisch für positive Scotome und demnach für Netzhautkrankheiten

*) Physiologie der Netzhaut pg. 142 u. f. und Handbuch von Graefe-Saemisch II. pg. 532. 
überhaupt angesehen werden dürfen, nämlich erstens $\mathrm{Er}$ haltensein der Empfindung für Roth bei Fehlen derselben für Blau und Grün, und zweitens Verwechslung von Blau mit Grün. Auch wird so der oben besprochene Unterschied der Bull'schen und der Heidelberger Pigmente verständlich. Die ersteren sind nämlich heller, weniger saturixt als die letzteren, und das Bull'sehe Blau hat schon bei Tageslicht einen deutlichen Stich ins Grunliche. Das pon mir angewandte Heidelberger Grün ist sehr hell (Grün N.2), das Blau dagegen saturirt. (Ultramarinblau N.2).

Eine Abnahme der Beleuchtung allein könnte demnach die Verwechslung dieses Blau mit Grün nicht erklären, da nach Aubert Dunkelblau bei abnehmender Helligkeit graublau erscheint*)

Die Eigenfarbe der getrübten Netzhautparthie hat neben der Beschattung einen sehr wesentlichen Einfluss auf die Entstehung der Anomalien des Farbensinnes bei positiven Sootomen.

In dieser Hinsicht sind an erster Stelle Blutangen za nemnen. Leber**) giebt an, dass bei diesen Blau grünlich

*) Hochecker (Gr. Arch. XIX. 3, pg. 32) hat jedoch bei mehreren normalen Personen gefunden, dass Cyan- und Indigblat bei geringen Helligkeitsgraden als grünlichgrau bezelehnet warden. Auch das von mir benutzte Heidelberger. Blau erschien einem

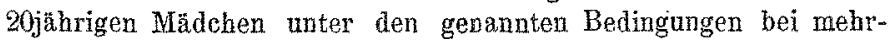
mals wiederholten Untersuchungen grünlich; ich selbst sehe dieselhen Veränderungen, wie sie Aubert bescbreibt. Es scheinen demnach individuelle Verschiedenbeiten vorzukommen. Für die Bull'schen Pigmente dagegen fand ich bei mir ebenso wie bei mehreren anderen normalen Individuen, dass verringerte Helligleit die Fähigkeit, Blau und Grin zu unterscheiden, authebt. Diese Verwechslung tritt bei dea schwächern Nuancen (N.1-6) viel leichter als bei den mehrgesättigten auf. Gleichzeitig möchte ich bemerken, dass ich das Bull'sche Roth (z. B. N. 18) ebenso wie mein Heidelberger bei kleinerem Gesichtswinkel und geringerer Helligkeit als Grïn und Blan im richtigen Tone erkenne.

**) Handb. von Graefe-Saemiseh V. pg. 1042. 
schwarz erscheint. Es ist jedenfalls bemerkenswertb, dass unter meinen acht Fällen, die das Heidelberger Blau als grünlich bezeiohneten, vier mit Blutungen an der Macula verzeichnet sind. Die meisten entzündlichen Exsudate haben eine gelbliche oder röthlich-gelbe Färbung, und oben ist ein solches von einer ausgesprochen grünlichen Farbe angeführt.

Auf Absorption von Seiten farbiger Netzhauttrübungen beruht auch wahrscheinlich die schon genannte, übrigens seltene Erscheinung, dass Hellgrün grau oder wejsslich erscheint, während Dunkelblau noch erkannt wird*).

Dass bei positiven Scotomen Blau mit: Grün verwechselt wird, hat meines Wissens Mauthner**) zuerst beobachtet and zwar bei jeder Form ron Erkrankung der Macula, sei es, dass der primäre Sitz in der Netzhaut, wie bei retinitis ex morbo Brightii, sei es, dass er in der Aderhaut sich findet, und das Leiden von der Chorioidea auf die Netzhaut übergegriffen hat***).

*) Eine Opticusatrophie ist ausgeschlossen, da Grün bei Rückgang des Leidens wieder erkannt wird. Verlust von Grün allein wurde viermal beobachtet, zweimal bei Blutungen.

**) Ueber farbige Schatten, Farbenproben und erworbene Erytrochloropie, Wiener med. Wochenschrift 1881 N.38 und 39.

***) Mauthner sieht meiner Ansicht nach mit Recht in der genannten Farbensinnstörung eine tür Netzhautkrankheiten charakteristische Erscheinung. Darin vermag ich ihm jedoch nicht beizum pflichten, dass es sich hiex um eine, vollkommene Erythrochloropie", eine "wahre Gelbblaublindheit" handelt, d. h., wenn ich ihn recht verstehe, um eine Erscheinung, die der angeborenen Erythrochlorople gleich zu setzen ist. Die erworbenen und die angeborenen Anomalien des Farbensimes sind meiner Meinung nach durchaus von einander verschieden, schon allein ans dem Grunde, weil nur bei diesen die Empfindung fur die in richtigem Tone erkannten Pigmente vollkommen normal ist, während bei jenen die richtig benanuten Pigmente nicht mels unter demselben kleinen Gesichtswinkel oder in derselben schwachen Nüance wie vom gesunden Auge wahrgenommen werden. Diese Verhältnisse können besonders deutlich nachgewiesen werden, wenn das zweite Ange intalkt ist, und gelten für Retinal- wie für Sehnervenkrankheiten. Daza 
Später hat Ole Bull*) mit Hilfe seiner Farbentafel umfassende Untersuchungen bei Retinalkrankheiten angestellt. Mit den Ergebnissen derselben stimmen die meinigen überein. Die Erklärung Bull's, der zu. Folge in der Schwăchung des Lichtsinnes, wie sie bei diesen Processen rorkommt, dje wesentlichste Ursache für die Storrungen des Farbensinnes beruhen soll, kann ich nicht als richtig anerkennen; denn die Anomalien des Farbensinnes in Folge ron Hemeralopie**) sind ron den in Rede stehenden durchaus verschieden. Wean Bull zur Begründung seiner Ansicht darauf hinweist, dass die Farbenverwechslungen bei Netzhautkrankheiten im Wesentlichen dieselben sind, welche bei einer unzureichenden Beleuchtung auch wom normalen Auge begangen werden, so kann ich diesen Vergleich nur für zutreffend halten, vermag aber darin eine Stütze für seine Anschaumg nicht zu finden.

„Ob die Verwechslungen sehwäsherer Nüancen von Grun und Blau, wie sie Patienten begehen, welche an Trübung der brechenden Medien hinter der Cornea leiden,

kommt noch, das 3 bei Amblyopien die nicht erkannten Farben unter grösserem Gesichtswinkel oder in stärkerer Saturation richtig bezeichnet werden (wenigstens in bestimmten Stadien dex Krankheit). Ueberdies erschein den Patienten mit positivem centralen Scotom keineswegs nur Blan als grün, sondern auch Grün als blau. Die Beurlheilung des Farbensinns nach der Benennung der Pigmente ist aber bef den erworbenen Störungen des Fiarbensinnes vollkommen fehlexfrei. In der Mauthner'schen Publication werden Angaben tuber die zur Untersuchung angewandten Objecte und uber die Zahl der gepruften Fäle vermisst, sowie specielle Beobachtungen, welche die in Rede atehende, bis dahin unbekante Thatsache beweisen.

*) Bemertungen uber dex Farbenginu unter vexshiedenen physiol, und pathol. Verhultaisen. Graefe's Aroh. XXIX. 3. pg. $71-116$.

**) Ich glaube annehwen zu müssen, dass Bull eine Reduction des Lichtsinnes, wie es zur Zeit seiner Arbeit allgemein geschah, für gleichbedeutend wit Nachtblindheit hält. 
mit den bei den eben besprochenen Affectionen auftretenden Verwechslungen in gleiche Klasse zu setzen sind", hält der genannte Forscher für zweifelhaft und führt als einzigen Grund für diese Ansicht ąn, dass nach seinen Beobachtungen "derartige Patienten Gelb und Roth nie für dunkler als Grün und Blau ausgeben."

Die Aufgabe, die Bull den Patienten stellt, über Helligkeitsdifferenzen der Farben urtheilen zu sollen, ist meinor Ansicht axch zu schwierig, um sichere Resultate zu ergeben. Schon fur Gesunde ist es, wie allgemein bekannt, sehr schwer, ein Urtheil über die Helligkeit verschiedenfarbiger Pigmente abzugeben, und ich muss von mir bekennen, dass ich es nicht mit Sicherheit vermag. Um etwas ganz Anderes handelt es sich, in Folge ron kleinem Gesichtswinkel oder geringer Belenchtung farblose Pigmente nach ihrer Helligkeit zu ordnen.

Aber auch die thatsächlichen Ergebnisse meiner Untersuchungen bei Patienten mit trüben Medien stimmen mit denjenigen Bull's nicht tuberein. Sie zeigen erstens Verwechslungen der fraglichen Farben nicht allein bei den cchwächeren, sondern auch bei den saturirten Nüancen, und zweitens, dass gelegentlich Gelb und Roth für dunkler als Grün und Blau gebalten wird.

Herr G., 49 Jahre alt, leidet auf dem rechten Auge an abgelaufener Iritis mit feiner Pupillarmembran und Glaskörpertrübungen.

L. E. $S=1$. R. E. $S=5 / 18$.

Bull'sche Tafel in 1 Meter:

L. N. 2 richtig, N, 1,5 Farben nicht erkannt*).

R. N. 18 Blau = Grün, ebenso bis incl. N. $2 ; \mathrm{N} 1, \check{a}$ keine Farbe.

Herr จ. P., 48 Jahre alt: diffuse Glaskörperträbungen nach iridocyclitis. 0 . d.

*) Die Beobachtung, dass die Farben von Bull N. 1 und 1,5 von normalen Augen nicht mehr erkannt werden, habe ich mehrfach genacht. 
S olne Glas $<5 / 36$, mit $+1 \mathrm{D}<5 / 18$.

Bull'sche Tafel in 1 Meter:

N. 18 Blau = Grün; N.3 ebenso; Roth und Gelb richtig; die letzteren erscheinen dunkler als Grün, auch sehon in N. 18.

Die Anomalien des Rarbensinns bei Trubungen der brechenden Medien sprechen demnach für meine oben dargelegte Erklärung der Störungen des Farbensinnes bei positiven Scotomen.

Es wäre daran za denken, dass ausser den beiden genannten Momenten die Hemeralopie bei der Entstehung der Functionsstorungen und speciell derjenigen des Farbensinnes der positiven Scotome eine Rolle spielt. Die. Möglichkeit hierfür ist bei einem Theil der letzteren, bei denen überhaupt Nachtblindheit beobachtet wird, insofern gegeben, als meiner Anschaung nach die Macula schon bei Tageslicht beschattet ist.

Es giebt nur eine für Hemeralopie pathognomonische Erscheinung, nämlich die qualitativen Anomalien des Farbensinnes bei herabgesetzter Beleuchtung, wie sie. von Förster zuerst beobachtet worden sind. Alle anderen Arten der Functionsstörungen der Hemeralopen bei geringen Helligkeitsgraden kommen auch bei Amblyopien zur Beobachtung \%). Diese qualitativen Farbenstörungen treten, so viel ich gesehen habe, bei positiven Scotomen nicht auf, wenigstens nicht bei Tageslicht. $O b$ der Grund hierfür darin zu. suchen ist, dass die Verdunklung in Folge der Retinatrübung nicht erheblich genug ist, oder dass - was ich fur wahrscheinlicher halte - die durch die (farbige) Trübung bedingten Anomalien des Farbensinnes diejenigen in Folge der Hemeralopie übertönen, dürfte nicht sicher entschieden werden können.

*) Ob die Störungen des peripheren Sehens bei Hemeralopen charakteristische Merkmale darbieten, ist bis jetzt nicht festgestellt. 
Es entsteht jetzt die wichtige Frage, welchen Einfluss Veränderungen der percipirenden Elemente auf die Entstehung der Schwachsichtigkeit bei positiven Scotomen ausüben. Man hat es bisher für selbstverständlich gehalten, dass die Abnahme des Gesichtssinnes bei Netzhautkrankheiten auf einer Affection der Stäbchen und Zapfen beruht, ebenso wie bei Sehnervenleiden auf einer solchen der Sehnervenfasern.

Wenn man hierin die einzige oder auch nur die wesentlichste Ursache der Selstörung bei Netzhautkrankheiten sieht und ebenso wie bei den Erkrankungen des Sehnerven eine mit dem Fortschreiten des Leidens zunehmende Herabsetzung der Erregbarkeit der percipirenden Elemente annimmt, so dürfte eine derartige Anschauung mit den Ergebnissen der Functionsprüfung, namentlich des Farbensinnes sohwer vereinbar sein.

Das charakteristische Merkmal der Farbensinnstörungen bei negativen Scotomen, überhaupt bei Sehnervenkrankheiten, - wie sie in allem Wesentlichen zuerst von Leber (Gr. Arch. XV. 3., pg. 26-107) in der klinisch bahnbrechenden Arbeit über das Vorkommen von Anomalien des Farbensinnes bei Krankheiten des Auges festgestellt und später von zahlreichen Untersuchungen bestätigt worden sind, - besteht bekanntlich darin, dass die Empfindung für gewisse Pigmente unter gleichem Gesichtswinkel in der Skala Grün-Roth-Blan abgesehwächt und schliesslich aufgehoben wird*).

*) Es scheint mir nothwendig zu sein, bei der Aufstellung des oben genannten Satzes hervorzuheben, dass die Pigmente nur dann in der bestimmten Reihenfolge verloren gehen, wenn sie unter gleichem Gesichtswinkel erscheinen. Ist dies nicht der Fall, ist z. B. das blane Object viel kleiner als das grüne, so kann ein Defect für Blan frither als für Grïn eintreten.

$O b$ sich ein Mangel der Empfindung für Grün oder Roth zuerst zeigt, hängt von den Nüancen der betreftenden Pigmente $a b ;$ im Allgemeinen verschwindet dasjenige zuerst, dessen Gesichtsfeld am kleinsten ist. 
Diese gesetzmässige Erscheinung wird damit erlulart, dass ein bestimmtes grünes Pigment ein schwächeres Reiznittel repräsentirt, als ein bestimmtes rothes, dieses ein schwächeres als ein blaues. Nimmt nun in Folge eines krankhaften Processes die Erregbarkeit der Sehnerrenfasern ab, so verliert sich zuerst die Empindung für Grün, dann für Roth und schliesslich fur Blau*).

Dem gegenüber wird bei positiven Scotomen bäufig Roth noch erkannt, wenn Blau grïn, seltener, wenn Blau farblos erecheint; und in negativer Hinsicht möchte ich anfübren, dass ich keinen Fall beobachtet habe, bei dem Blau allein in richtigem Tone wahrgenommen wurde.

Wenn man nun die oben genannte Erklärung der Farbensinnstörung bei Sehnervenlrankheiten als richtig anerkennt; und wenn man ferner annimmt, dass eine Herabsetzung der Frregbarkeit des nervösen Sehapparates stets dieselben Anomalien des Farbensimes hervorrufen muss, gleichriel ob sie durch eine Affection der Sehnervenfasern oder der percipirenden Netzhautelemente bedingt ist, so kommt man zu der Schlussfolgerung, dass bei positiven Scotomen eine Abnahme der Erregbarkeit der percipirenden Elemente entweder überhaupt nicht erfolgt oder ihren Einfluss auf die Erscheinungen des Farbensinnes gegenüber anderen Momenten (farbige Netzhauttrübung) nicht geltend zu machen im Stande ist.

Die Frage nach dem Einflusse einer Affection der peroipirenden Elemente auf die Entstehung von Amblyopie bleibt demnach eine offene**).

*) Diese von Schön (Die Lehre vom Gesichtsfelde, Berlin, 1874) aufgestellte, dann von mir (Gr. Arch. XXV. 2. u. 3) verfochtene Anschauung scheint sich, wie die Literatur der letzten Jahre zeigt, einer allgemeinen Zustimmang zo erfreuen.

**; Klinisch kennen wir zwei Functionsstörungen, die sicher durch eine Anomalie der Stäbchen and Zapfen hervorgerufen werden können, das sind absolute Amaurose und Metamorphopsie; anatomische Untersuchungen kranker Netzhäute haben dem ent- 
Die Trübung der Netzhautsubstanz ist das einzige Moment, das wir bei dem jetzigen Stande unserer Kenntnisse sicher als eine Ursache der Sehstoorung bei positiven Scotomen bezeichnen dürfen. Dieselbe ist ophthalmoskopisch and pathologisch anatomisch festgestellt und erklärt die Functionsstörungen ungezwungen und zugleich so vollständig, dass wir sie schon jetzt als das wesentlichste Moment für die in Rede stehende Frage ansehen dürfen.

Hiermit ist ein durchgreifender Unterschied in der Entstehung der Anomalien des Gesichtssinnes bei Erkrankungen der Netzhaut und bei Erkrankungen des Sehnerven gegeben, und die im Eingang dieser Abhandlung aufgeworfene Frage beantwortet, warum nur bei Affectionen des Leitungsapparates die Empfindung für die Pig-

sprechend vollkommenen Verlust und Lageveränderungen der genannten Elemente nachgewiesen. Die letztere Affection braucht an sich gar keine oder nur sehr geringe Schwachsichtigkeit zu bewirken, wie Fälle von ausgesprochener Metamorphopsie resp. Micropsie und Macropsie mit fast normaler Sehschärfe zeigen. Die pathologische Anatomie hat uns ausserdem Formveränderungen der Stäbchen und Zapfen kennen gelehrt; ob diesen stets Sehstörungen korrespondiren und welcher Art sie sind, ist bis jetzt nicht bekannt.

Der Einfluss pathologischer Veränderungen der percipirenden Elemente auf das Zustandekommen von Functionsstörungen wird uns wohl nicht früher verständlich werden, bis ihre Rolle für die Physiologie der Gesichtsempfindungen klar gelegt ist.

Mir kommt es nur darauf an, auf unsere Unkenntniss bezüglich einer Affection der Stäbchen und Zapfen als Ursache von Amblyopie die Aufmerksamkeit mehr, als es bisher geschehen, hinzulenken; es liegt mir aber vollkommen fern, die bedeutung dieser für das normale Sehen sehr wichtigen Gebilde für die Pathologie leugnen $z u$ wollen. Wenn in den percipirenden Elementen sog. Sehstoffe enthalten sein sollten, die den Umsatz von Aetherbewegung in Nervenreiz vermitteln, so würde die Pathologie der genannten Elemente mehr auf chemischen, als auf anatomischem Gebiete festzustellen sein. 
mente in der mehrfach genannten Reihenfolge abgeschwächt wird.

Für die hier vorgetragenen Anschauungen scheinen mir Beobachtungen von solchen cireumscripten Blutungen am hinteren Pol sehr wichtig zu sein, die, wie ophthalmoskopisch sicher diagnosticirt werden kann*), an der Innenflache oder in den inneren Schichten der Retina ihrea Sitz haben.

Sie sprechen erstens für die Erklärung des positiven Scotoms als eines entoptischen Phänomens. Einerseits kann Hemeralopie hier nicht in Betracht kommen, da sie nicht vorhanden ist. Andererseits unterliegt es keinem Zweifel, dass die Blutung einen Schatten auf die hinteren Schichten dex Retina werfen, und dass dieser Schatten ron den Patienteu wahrgenommen werden muss, Entsprieht doch auch die Grösse and Form des Sootoms demjenigen der Blutung.

Die fraglichen Falle sprechen zweitens fur die $\mathrm{Be}-$ deutung der Netzhauttrübung als Ursache von Schwachsichtigkeit; denn eine irgend erhebliche Affeetion der percipirenden Elemente scheint mir wegen der Lage der Blutung angeschlossen su sem. "Diese Auffassung wird auch dadurch bewiesen, dass die Besserung des Sehvermögens mit der Aufsaugung des Blutes Hand in Hand geht, dass ferner das Scotom um so heller erscheint, je mehr das Blat abnimmt, und dass endlich gleichzeitig mit dem Verschwinden des Schattens die Sehschäre sich erheblich bessert resp. normal wird**). bis 554 .

*) of. Leber im Handh. von Graefe-Saemisch V. pg. 552

冰脐) Die entsprechenden Erscheinungen werden bei den positiven Scotomen in Folge von anderen Krankheiten beobachtet; im Allgeneinen entspricht die Amblyopie den sichtbaren Veränderungen. der Macula lutea und eine Zunabme des Sehvermögens der Rückbildung der Netzhauttrübung. Bemerkenswerth erscheint es auch, dass die Kranken das positive Scotom noch bei äsuerst gexinger Sehschärfe wahrzanehmen im Stande sind. 
Die Beobachtung solcher Falle erscheint deshalb so wichtig, weil die Sehschärfe dabei sehr erheblich, bis auf $1 / 20$ und mehr, herabgesetzt sein kann. Denn damit ist der Beweis geliefert, dass sehr hochgradige Functionsstörungen ohne irgend wesentliche Affection der Stäbchen und Zapfen, allein in Folge von Abnahme der Durchsichtigkeit des Retinagewebes zu Stande kommen können.

In demselben Sinne argumentiren Fälle ron markhaltigen Sehnervenfasern, bei denen, soviel mir bekannt, die anatomischen Untersuchungen Veränderungen der hinteren Netzhautschichten nicht gezeigt haben. Drei Patienten mit dieser Anomalie habe ich am Perimeter genau geprüft. Von zweien wurden die Farben der $20 \mathrm{Mm}$. Quadrate in dem der kranken Netzhautparthie entsprechenden Abschnitt des Gesichtsfeldes richtig, nur als beschattet, vom Dritten als grau bezeichnet*); dabei war der Farbensinn im Uebrigen vollkommen normal. In einem von Hirschberg**) beschriebenen Fall von markhaltigen Sehnervenfasern im Netzhautcentrum war $\mathrm{S}=$ Finger in 1 Fuss.

Wenn diese hochgradigeSehschwäche auch wabrseheinlich nicht allein auf das dioptrische Hinderniss zurückzuführen ist, sondern ausserdem auf amblyopia congenita resp. ex anopsia, so darf man doch annehmen, dass sie wesentlich von denselben beeinflusst worden ist. -

Es kann wohl keinem Zweifel unterliegen, dass die Sehstörung in den übrigen Theilen der Netzhaut in derselben Weise zu Stande kommt wie an der Macula lutea.

Die Entstehung der peripheren positiven Scotome, die bei disseminirter chorioretinitis in erheblicher Zahl und

*) In solchen Fallen von einer Verbreiterung des blinden Flecks zu sprechen, ist nicht vollkommen richtig, da nur im blinden Fleck selbst die Empfindung ganz fehlt.

**) Klinische Beobachtungen aus der Augenheilanstalt. 1874. pg. $66-68$. 
geringer Ausdehnung, bei amotio retinae in grôssereme Umfang auftreten, ist ebenso wie die der centralen zu erklären. Bei denselben wird ebentalls die charakteristische Verwechslung von Blau und Grün beobachtet.*)

Abgesehen ron diesen Defekten sind die Aussengrenze und die Farbenfelder bei den verschiedenen Formen von Retinitis und Chorioretinitis der Regel nach normal. Diese Erscheinung stimmt mit der ophthalmoskopisch and pathologisch anatomisch nachgewiesenen Thatsache überein, dass die Veränderungen bei den diffusen Entzündungen der Retina nach der Peripherie des Augengrundes erheblich abnehmen: die Netzhauttrübung ist daselbst zu gering, urn Veränderungen des Farbensinnes herbeizuführen. Andererseits folgt meiner Ansicht nach aus dem Verhalten der Farbenfelder, dass die \&rregbarkeit der Sehnervenfasern der Retina durch die entzündlichen Processe dieser Membran garnicht oder um eine so geringe Quote herabgesetzt wird, dass mit den gewöhnlich angewandten Untersuchungsobjecten ein Defect nicht arkannt wird. Anderenfalls müssten ähnliche Störangen des peripheren Farbensinnes bei Netzhautkrankheiten beobachtet werden wie bei Sehnervenleiden. $*$ *

Zum Schluss möchte ich bezüglich der erworbenen

*) Sie ist bei den peripheren Seotomen in Folge von amotio retinae länger als bei den centralen bekannt, lueber (Handb. von Graefe-8aemiseh, V. pg. 690) hat die ersten Mittheilungen dariber, danach Cohn (Centrabl. \& Angenh. $1877 \mathrm{pg} .146$ ) einen derartigen Fall veröfentlicht; später ist von mir (l. c.) auf das bäufige - Vorkommen dieser Anomalie aufmerksam gemacht und gezeigt worden, dass sie auf den der Ablosnng entsprechenden Theil des Gesichtsfeldes beschränkt sein kan. In nenester Zeit bat Bull (l. c.) diese eigenthumliche Perversion des Farbensinns bei allen Netzhautkrankheiten festgestellt.

* A w einer Afektion der Nervenfasern der Retina beruhen sectorenfôrmige Defecte, die bekanntlich dam entstehen, wenu die Leitung in einem Bändel you Nervenfasern (meist in Folge von Blutung) unterbrochen wird. 
Farbenblindheit*) noch auf folgende zwei Punkte hinzuweisen mir erlauben.

Wir pflegen unser Urtheil über den Farbensinn von dem Verhalten desselben in der Gegend der Fovea centralis abhängig zu machen, abgesehen von centralen Scotomen, deren Vorhandensein wohl immer besonders hervorgehoben wird. Gegen dies Vorgehen lässt sich um so weniger ein Einwand erheben, als wir bei der Bezeichnung des Lichtsinnes und der Sehschärfe nicht anders verfahren. Es erscheint aher doch nicht überflüssig, darauf hinzuweisen, dass dann aus dem Verhalten des Farbensinnes auch nur auf die Functionstüchtigkeit der die Fovea**) und deren Umgebung innervirenden Sehnervenfasern resp. deren Endigungs- und Ursprungsstelle geschlossen werden darf.

Der Gebrauch, das Verhalten des centralen Farbensinnes als massgebend für dasjenige des Farbensinnes uberhaupt anzusehen, hat einen wesentlichen Einfluss bei der Aufstellung des wohl kaum angefochtenen Satzes ausgeübt, dass der erworbene Verlust des Farbensinnes ein pathogno-

*) Störungen des Farbensinnes ebenso wie des Raum- und Lichtsinnes können abgesehen von Trübungen der brechenden Medien in dreifacher Weise entstehen: durch Affektion der Sehnervenfasern, durch Opacität der Netzhaut und durch Hemeralopie (Störung der Adaptation in Folge von mangelhafter Production des Sehpurpurs).

**) Becker (Neue Untersuchungen über excentrische Sehschärfe und ihre Abgrenzung von der centrischen, Dissertat., Halle 1883) hat in der Graefe'schen Klinik die wichtige Thatsache festgestellt, dsss ganz vormale Sehschärfe nur in einer Gesichtsfeldausdehnung von $1 \frac{1 / 2}{}{ }^{0}$ besteht, dass also die periphere Sehschärfe schon in der Macula lutea, neben der fovea centralis, beginnt. Hierin sehe ich eine Bestätigung meiner früher ansgesprochenen Ansicht (Gr. Arch. XXV. 2, pg. 83), dass der centrale Farbensinn ungefähr einem Gesichtswinkel ron $1^{\circ} 13^{\prime} 22^{\prime \prime}$ entspricht, dass bei Quadraten von $20 \mathrm{Mm}$. Seite in 1 ' Entfernung, die unter einem Gesichtswinkel von $3^{\circ} 38^{\prime} 59^{\prime \prime}$ erscheinen, der Einfluss des peripheren Farbensinnes schon sehr erheblich ist. 
monisches Merkmal der Atrophia n. opt. ist. Der Satz hat in dieser Allgemeinheit nur deswegen Gültigkeit, weil es im Wesen der Opticusatrophie liegt, allmählich alle Nervenfasern, einschliesslich derjenigen der Macula lutea zu ergreifen; daher erseheint auch der von Schön vorgeschlagene Name "Totalatrophie $e^{i b}$ sehr zweckmässig.

Thatsächlich erfolgt ein Verlust des Farbensinnes auch bei den Partialatrophien und bei den Netzhautkrankheiten (abgesehen von secundärer Atrophie der Sehnervenfasern), so dass alle Pigmente farblos erscheinen. Die Differenz gegenttber der Totalatrophie zeigt sich darin, dass die Anomalie des Farbensinnes sich nur ausnahmsweise auf das ganze Gesichtsfeld*) verbreitet, dass der centrale Farbensinn oft normal bleibt. Der principielle Unterschied zwischen Sehnerven - und Netzhautkrankheiten besteht nicht in dem Verlust des Farbensinnes, der beiden zukommt, sondern in der Art and Weise, in der sich dieser Verlust entwickelt.

Zweitens möchte ich darauf hinweisen, dass die gebräuchliche Untersuchungsmetbode mit Heidelberger Pigmenten in Bezug auf die Anomalien des Farbensinnes nur relative Schlüsse, d. h. für einen Vergleich der verschiedenen Arbblyopien unter einander, aber keine absoluten für den speciellen Fall gestattet. Wenn Quadrate von 20 oder $10 \mathrm{Mm}$. Seite in Perimeterdistanz central richtig bezeichnet werden, so ist damit nicht ein normaler Farbonsinn nachgewiesen; er kann trotzdem nicht unerheblich herabgesetzt sein**). Ein achtet.

*) Diese Eirscheinung wird bei totaler amotio retinae beob-

**) Diese Behauptung wird durch die oben mitgetheilten Krankheitsgeschichten bewiesen; sie zeigen erhebliche Störungen des Farbensinnes an der Ball'schen Tafel, selbst wenn kleine Heidelberger Quadrate ron $2 \mathrm{Mm}$. Seite in $1^{\prime}$ Entfernung richtig benamnt werden "Gleiche Beobachtungen habe ich bei Sehnervenleiden und bei Glaucom gernacht. - $\mathrm{Zu}$ den Grenzbestim- 
ganz einwandsfreies Urtheil über normales Verhalten des Farbensinns kann nur dann abgegeben werden, wenn die Pigmente in derselben Entfernung wie vom gesunden Auge erkannt werden, wenn der Nachweis einer vollkommenen Uebereinstimmung mit dem Verhalten des gesunden Auges

mungen wird meiner Ansicht nach die Bull'sche Tafel besser angewandt, als die Methode, den kleinsten Gesichtswinkel festzustellen, unter dem saturirtere Pigmente im richtigen Tone erkannt werden. Diese Untersuchung ergiebt keine ganz eindeutigen Resultate, weil benachbarte Theile des nervösen Sehapparates als gleichwerthig betrachtet werden, während sie thatsächlich in verschiedenem Grade afficirt sein können. Die Bull'sche Tafel gestattet ohne erheblichen Zeitaufwand eine numerische Bestimmung des centralen Farbensinnes und erfüllt in dieser Hinsicht ihren Zweck. Dass bei derselben die normale Grenze zu gering gesetzt ist, habe ich schon oben erwähnt; nach meinen Beobachtungen kann N.2 als Norm angenommen werden. Auch darauf ist schon aufmerksam gemacht worden, dass die für Netzhautkrankheiten charakteristische Verwechslung von Grün und Blau bei den Bull'schen Pigmenten leicht zu Tage tritt. Dasselbe ist aber wahrscheinlich auch bei hellgrünen und hellblauen Heidelherger Pigmenten der Fall.

Bull (l. c.) glaubt mit Hilfe seiner Farbentafel gefunden zu haben, dass bei gewissen Formen von Opticusatrophie eine Schwäche nur für die complementären Farben Roth und Grün, bei anderen für alle Farben besteht, und zwar soll die erste Art der Farbensinnstörung charakteristisch sein für ein ,primäres Leiden im Innern des Centralnervensystems, durch welches die ästhesodischen Ursprungszellen des Sehnerven afficirt werden", "für ein primäres Leiden der Neuraxe" (?). Diese Untersuchungen lassen sich in den Rahmen der bisher bei Opticusatrophie festgestellten Anomalien des Farbensinnes einfügen: die Fälle mit Roth-Grünblindheit stellen meiner Ansicht nach ein frühzeitiges Stadium der Opticusatrophie dar, in dem die Empfindung für Blau noch wenig gelitten hat. Einer derartigen Auffassung widersprechen die von B ull angeführten Fälle nicht, da sich in keinem die Empfindung für Roth und Grün bei ganz normaler für Blau und Gelb sehr erheblich geschwächt zeigt. Die Empfindung für das Bull'sche Roth und Grün muss nach unserer Auffassung der Entstehung der Anomalien des Farbensinnes bei der Opticusatrophie stets gleichmässig geschwächt sein, da beide vom normalen Auge gleich weit nach der Peripherie erkannt werden, also gleich starke Reizmittel darstellen. 
Dr. Th. Treitel.

geliefert ist. Andererseits folgt ans dem Verlust der Empfindung für die Heidelberger Pigmente ebensowenig wie aus dem fur eine bestimmte Nüance der Bull'schen Pigmente eine absolute Farbenblindheit. Weder die eine noch die andere Untersuchung gestattet den Schluss, dass stärker saturirte Pigmente bei gleichem, dieselben Pigmente bei grösserem Gesichtswinkel nicht richtig erkannt werden; beide Male handelt es sich, ebenso wie bei der Einschränkung der peripheren Farbengrenzen und bei der physiologisch farbenblinden Zone, nur um relative Verhältnisse.

Königsberg i. Pr, im Februar 1885. 